\title{
SOCIAL DISTANCING AND MASK DETECTOR BASED ON COMPUTER VISION USING DEEP LEARNING METHODS
}

\author{
Sunil Bhutada, Nirupama, N. Sahithi, M. Mounika and M. Revathi \\ Department of Information Technology, Sreenidhi Institue of Science and \\ Technology, Ghatkesar, Hyderabad \\ sunilb@,sreenidhi.edu.in, 17311a1252@,sreenidhi.edu.in
}

\begin{abstract}
:
In the context of a coronavirus pandemic, this paper describes social distancing and face mask identification. In a pandemic such as this one, social distancing and adopting a face mask may help you feel better. The Covid-19 had a great impact on the health of aspects in a large number of countries, and so it brought a difficult time for a lot of people across the world. By limiting the transmission of infection, the effective measure of wearing a face mask and maintaining social distance can save billions of lives. In our project, we presented two main solutions. (i)Masking; (ii) In real-time, maintaining a secure (physical/social) distance between people. Features: (I)Face Masking: The camera can detect people who wear masks and that those who are not wearing masks, using green and red boxes denoting who is wearing masks and who's not. When the machine detects a human without a mask or facial covering, it generates an alarm. (II)Social Distancing: The appliance analyzes the distance between the two individuals and flashes red and green boxes, denoting that the red box is not retaining a healthy distance whereas the green box is. (III) Warning: An alarm is triggered if a person does not maintain distance and has no face covering; an alarm is triggered if a person does maintain the distance but has no face covering and an alarm is triggered if a person has a face hidden but has not retained distance.
\end{abstract}

Keywords: Cnn,computer-vision,deep learning,mobile net,open cv

\section{INTRODUCTION:}

Social isolation and wearing masks have become the two most prominent concepts in today's society to avoid being bitten by the novel coronavirus. The COVID warriors, according to researchers, are these two roles. Governments around the country are working hard to accomplish these kinds of policies. We designed a real-time methodology for analyzing human distance and identifying individuals who aren't wearing a mask. People are clueless about the most fundamental precautions, Mask+Distance, which needed this solution. Only the combination of the two, not only from the mask or the separation, can safeguard you. Computer Vision is a subset of Artificial Intelligence that tends to make use of a computer's processing capacity to collect relevant information from data of photographs, videos, and other media. Computer vision is used for a range of different purposes in this world on the use cases. Artificial Intelligence (AI) is a held name for technologies such as Machine Learning, Deep Learning, and Computer Vision. Face Mask Detection \& Social distancing is a research project that combines computer vision to identify various components of pictures or videos utilizing frames as input. Coronavirus has a massive effect on the country's major activities, including production, transportation, and 
agriculture. As a response to the consequences, the entire planet was driven to freeze all functions and implement severe controls for social distancing and wearing a face mask as a matter of necessity. Covid-19's influence on diverse fields is illustrated.

\section{METHODOLOGY:}

\section{Existing Solutions: Only Face mask detection Proposed :}

Convolutional neural networks (CNNs) are neural networks comprising one or more convolutional layers that are used to classify, predict, segment, and examine auto-correlated data. The process of sliding a filter upon an input signal is defined as convolution. OpenCV is a large open repository for computer vision, machine learning, and image recognition which nowadays plays a key role in real-time operations, which are crucial in today's systems. In photos and videos, it can recognize objects, individuals, and even human handwriting. Deep learning is a branch of the machine learning method which uses multiple layers to retrieve higher-level functionality from raw data. Lower image processing layers, for example, may specifically set, while higher layers may human dental concepts like numbers, words, or faces. Despite the reality that several faces, entity, landmark, emblem, and text recognition and detection technologies are available for Internet-connected devices, we expect that the improving processor ability of mobile devices will permit these technologies to be offered to clients anytime, despite Network access. On the other hand, on-device and embedded computer vision present serious risks: models must function fast and efficiently in a resource-constrained space despite utilizing the least computation, power, and energy. Tensorflow provides a range of pretrained models, such as drag-and-drop models, that can classify up to 1,000 items. MobileNet excels analogous models in terms of latency, expandability, and precision. In terms of production efficiency, a full-fledged model has a huge level of interruption.

There were four main huge obstacles to overcome.

i. Data Collection -We scraped data from the web (Web Scraping) and collected a few shots because no data set of individuals wearing masks were available.

ii. Training Data - As this site scraped data only delivered a few shots, we'd pick a pre-trained model like MobilenetV2.

iii. Integration of two very different modules While mask recognition just better reflects covering the face, social distancing takes into consideration two distances between individuals. When it comes to integration, we must concentrate on the following things at once:

(1) mask shielding and

(2) real-time distance estimation between two or more persons.

\section{iv. Calculation of Euclidean distance-}

We can get to the next stage now that we've successfully recognized the faces in each snapshot: determining the distance between them. In the other phrases, the distance between the centroid of each drawn rectangle should be computed. To execute so, we'll need to determine the ratio of pixels to centimeters $(\mathrm{cm})$ based on a known distance for the given object comparison. The reference pixels method generates this value, which is needed in the faces dist module:

We may use the basic Euclidean distance formula to compute the distance between each centroid, and then utilize the ratio to transfer it to measurement units $(\mathrm{cm})$ :

$\mathrm{D}=\operatorname{sqrt}(($ point $1 \mathrm{x}+$ point $2 \mathrm{x}) 2+($ point $1 \mathrm{y}+$ point2 y ) 2 )

Our annotate faces function adds the approximation to a line drawn from each centroid and uses this simple formula. The line will be red if the approximation is much less than the minimum size necessary. 
In our project, we have incorporated a face detection model and instead of Yolo, we are using mobilenet for preprocessing and training.

\section{RESULTS:}

Results are shown in Fig 4-11.

\section{Conclusion and Future scope:}

For the Covid-19 incidents, this research aimed to learn more about social distance and face mask identification. People were also used to detect objects for social distance, and faces were being used to detect surgical masks, all of which were accomplished with Opencv. The Opencv for target tracking was in duty of Darknet. The outputs for social distance were carried out on multiple sites of recordings to make it very difficult to identify. In contrast, overloaded places were assessed. The face mask tracking model displayed the \% accuracy for each object spotted. Large companies may implement this with real-time tracking, which would require extra computing resources. This technique is beneficial since it can be enforced leveraging IoT capabilities on-road cameras/malls/etc. to determine which venues are violating the rules and take the necessary action, while individuals who have unwittingly broken any laws may be notified with an alarm/alert at the moment of the infraction.

\section{REFERENCES:}

Howard AG, Zhu M, Chen B, Kalenichenko D, Wang W, Weyand T, Andreetto M, Adam H Mobilenets (2017): efficient convolutional neural networks for mobile vision applications. covid19.who.int. (n.d.). WHO coronavirus disease (COVID-19) dashboard.

. Redmon, S. Divvala, R. Girshick, and A. Farhadi, (2016) "You only look once: Unified, real-time object detection," in Proceedings of the IEEE conference on computer vision and pattern recognition.

O. Russakovsky, J. Deng, H. Su, J. Krause, S. Satheesh, S. Ma, Z. Huang, A. Karpathy, A. Khosla, M. Bernstein, et al., "Imagenet large scale visual recognition challenge," International journal of computer vision.

R. Girshick, J. Donahue, T. Darrell, and J. Malik, "Rich feature hierarchies for accurate object detection and semantic segmentation," in Proceedings of the IEEE conference on computer vision and pattern recognition, (2014)

David Oro ; Carles Fernández ; Javier Rodríguez Saeta ; Xavier Martorell ; Javier Hernando. Real-time GPU-based face detection in HD video streams.

F Sener, N Ikizler-Cinbis, Two-person interaction recognition via spatial multiple instance embedding

Yadav, Shashi. (2020) "Deep Learning-based Safe Social Distancing and Face Mask Detection in Public Areas for COVID-19 Safety Guidelines Adherence." International Journal for Research in Applied Science \& Engineering Technology (IJRASET).

Belciug, S., 2020. Artificial intelligence in cancer: Diagnostic to tailored treatment, London, United Kingdom. 
Fig.1- Pie-Chart for Impact Distribution

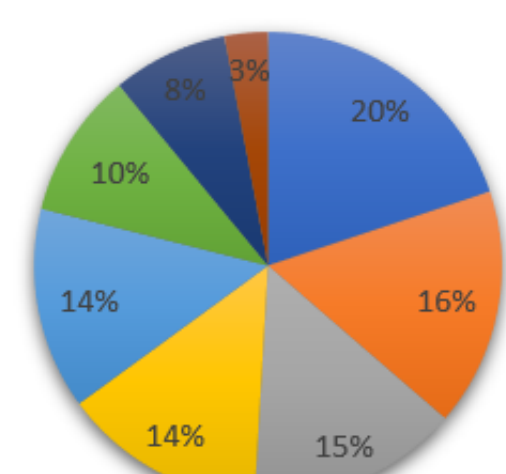
- Restaurants
neal Estate
Aviation
Infrastructure
- Trading
- Tourism
- Manufacturing
- Agriculture

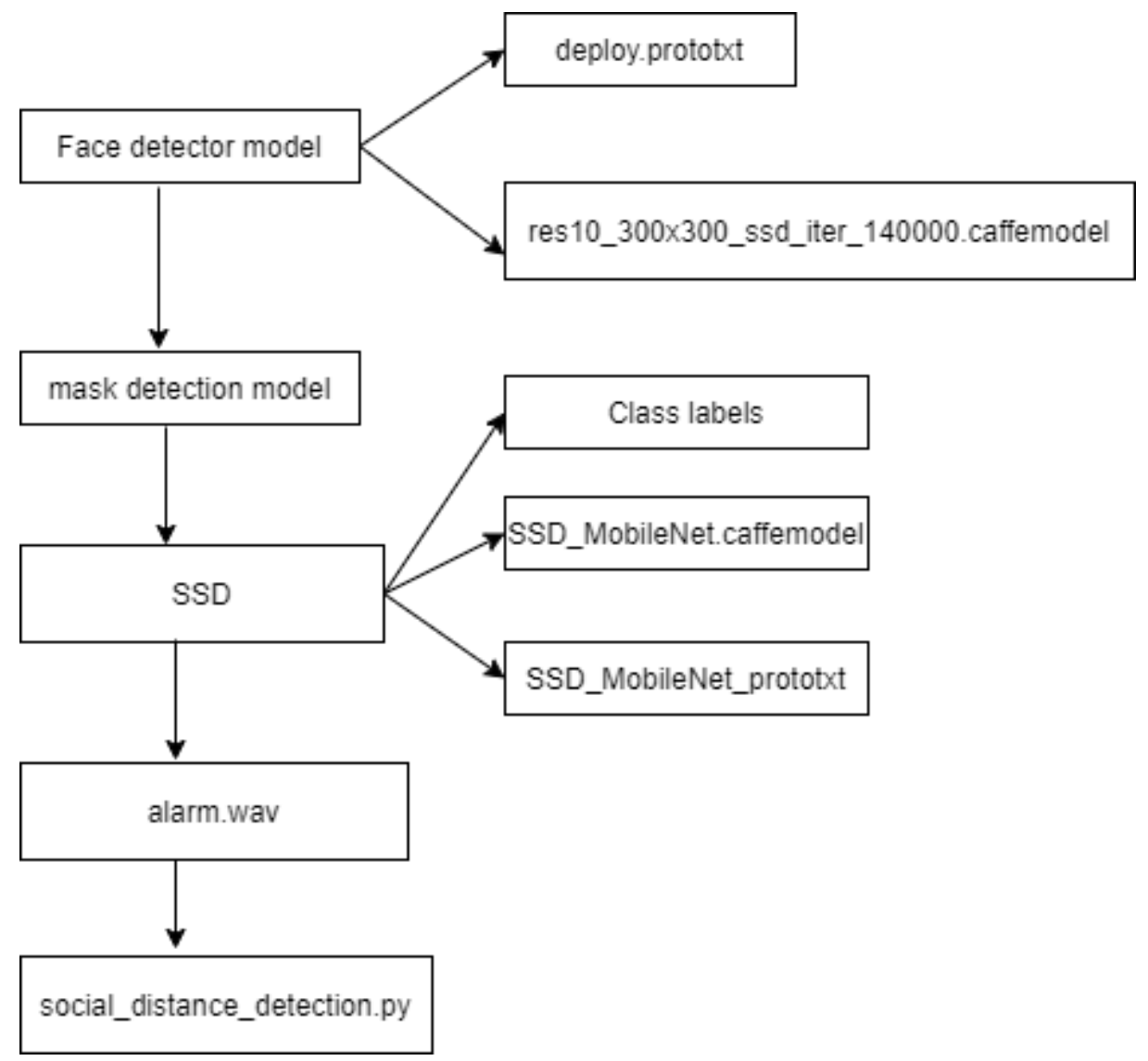


I J R B A T, Issue (IX), Vol. II, May 2021: 74-

A Double-Blind Peer Reviewed \& Refereed Journal
OPEN $\bigcirc$ ACCES

e-ISSN $2347-517 X$

Original Article

\section{$>c d$ C: \Program Files\mask_socdist_det}

Fig. 4 - Execution

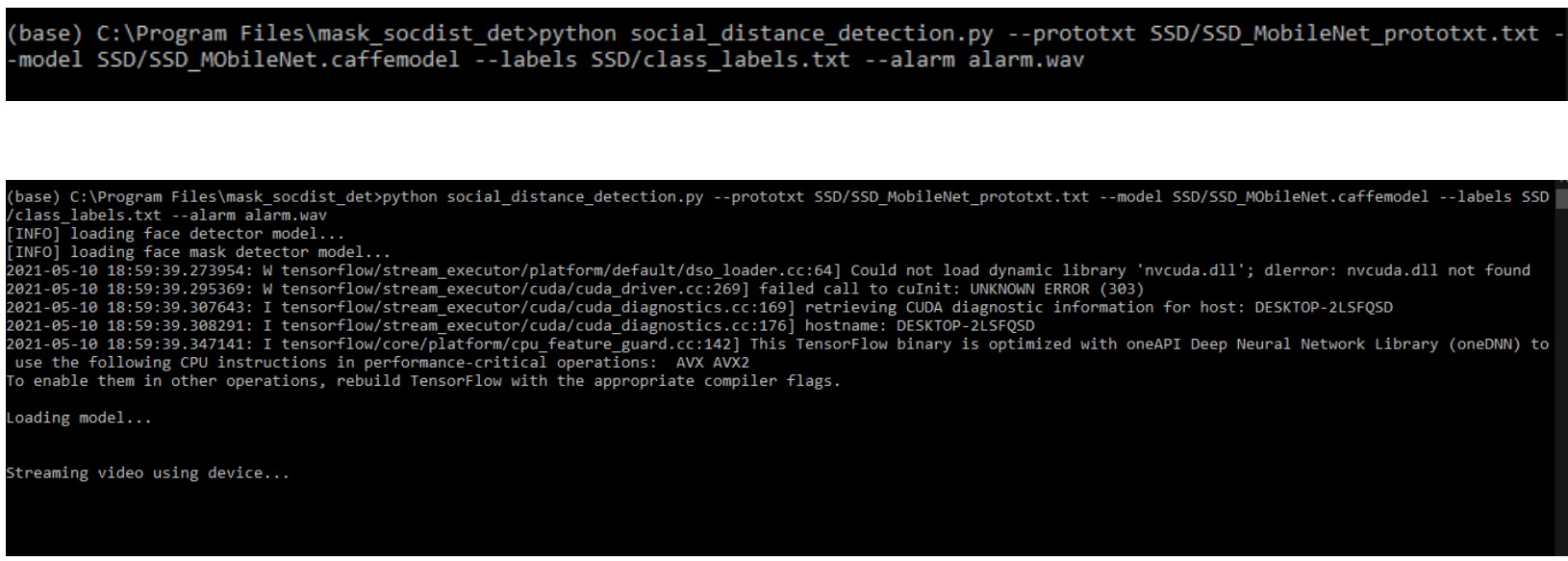

Fig. 5 - Front End

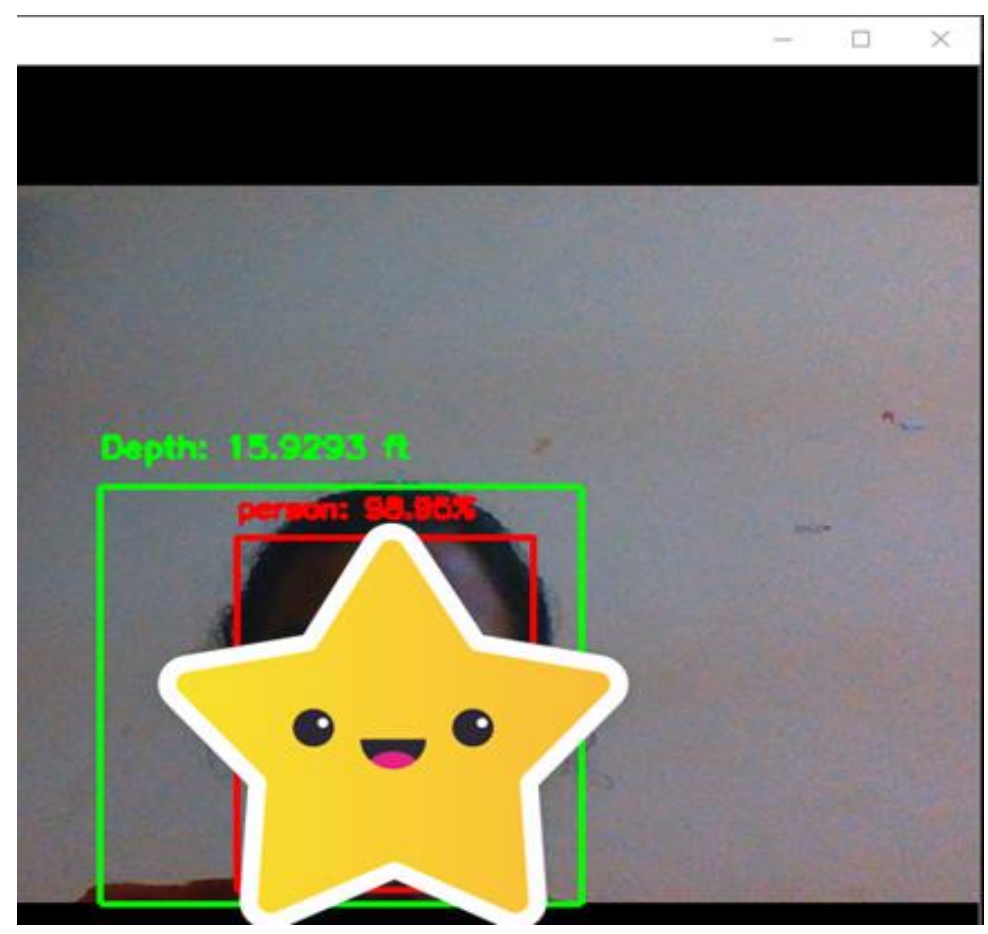

Fig.6 - Face Detection 


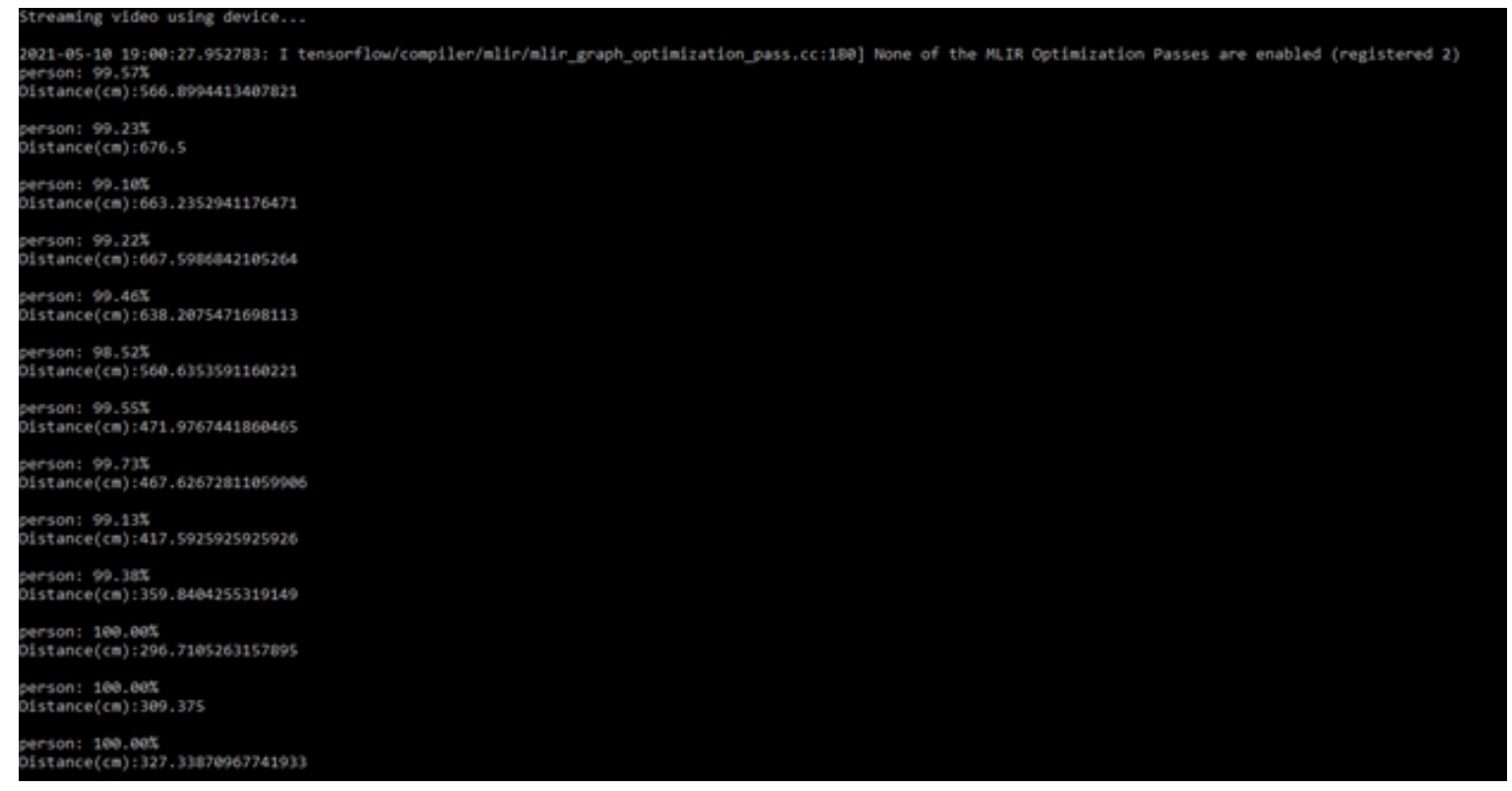

Fig. 7 - Face Detection accuracy and Distance Calculation

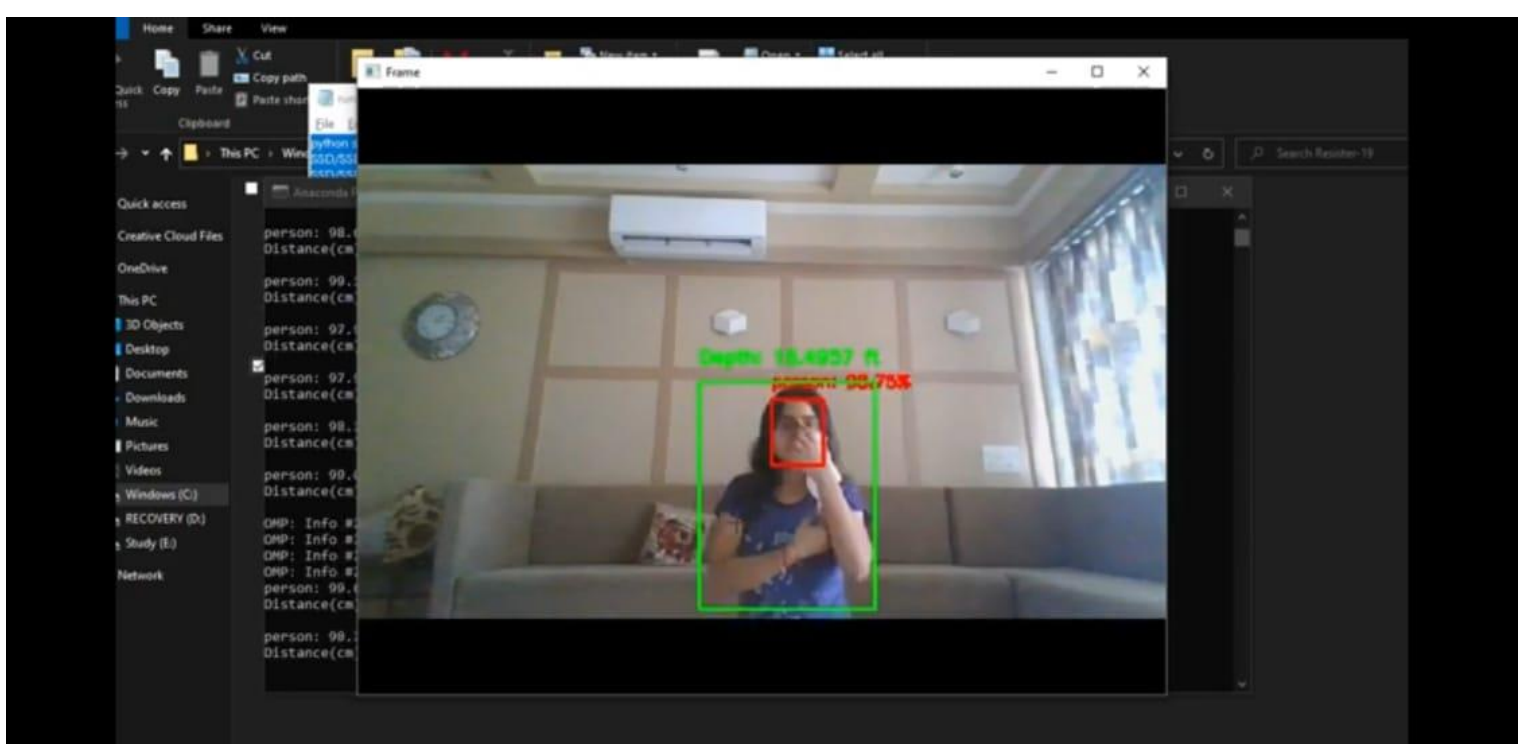

Fig.8 - Without mask - Alarm 


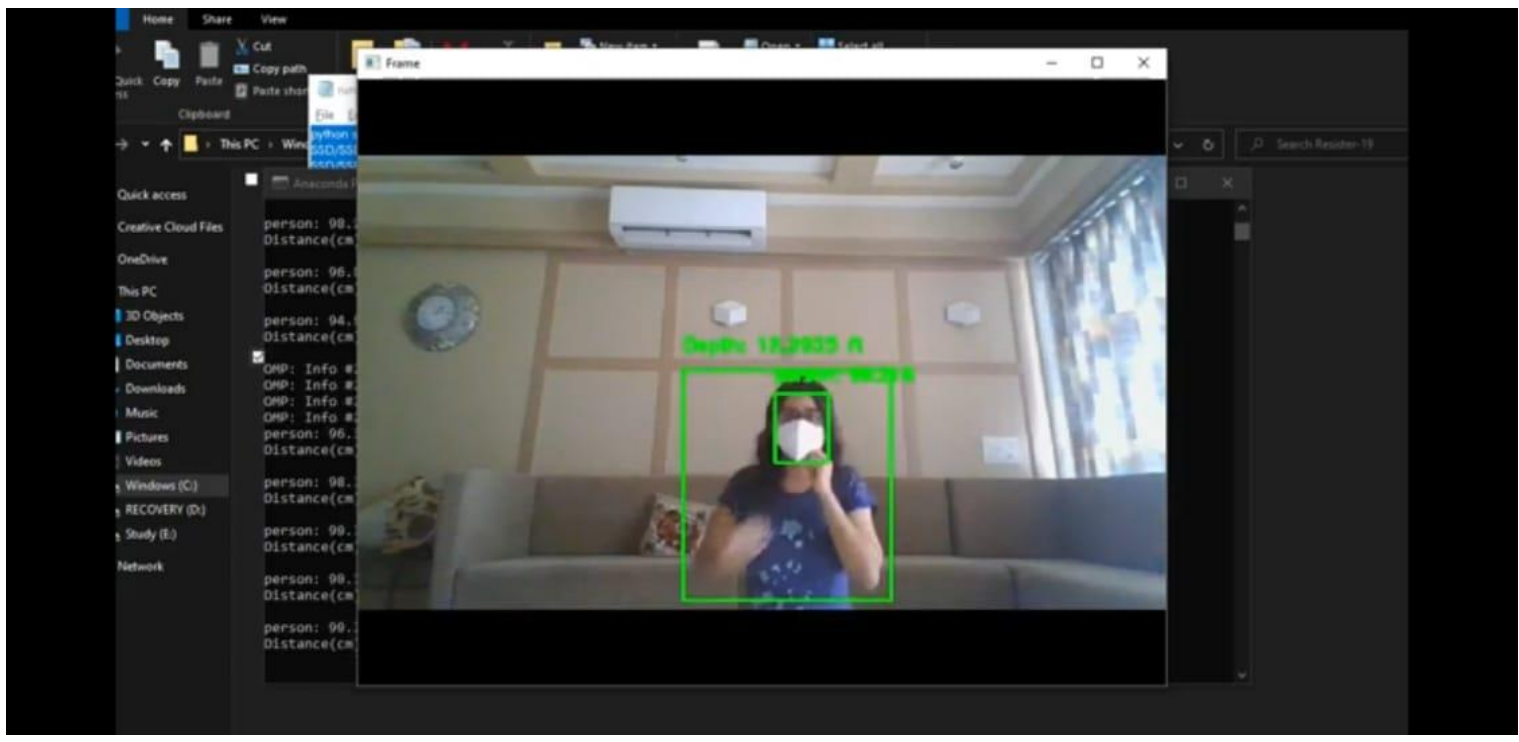

Fig.9 - With mask -NO alarm

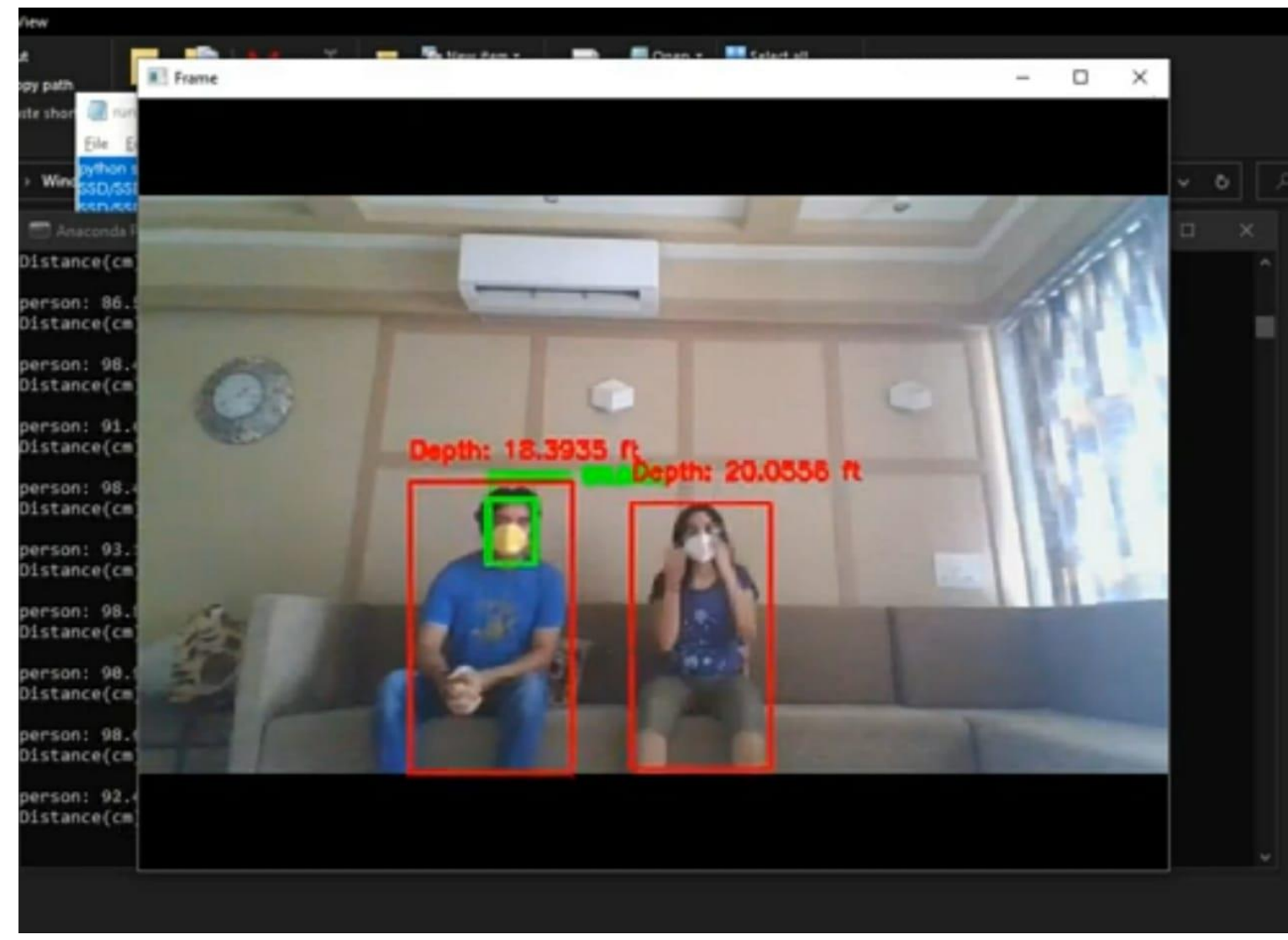

Fig.10 - Not maintaining social distance- Alarm 
I J R B A T, Issue (IX), Vol. II, May 2021: 74-

A Double-Blind Peer Reviewed \& Refereed Journal
OPEN $\bigcirc$ ACCES

e-ISSN $2347-517 X$

Original Article

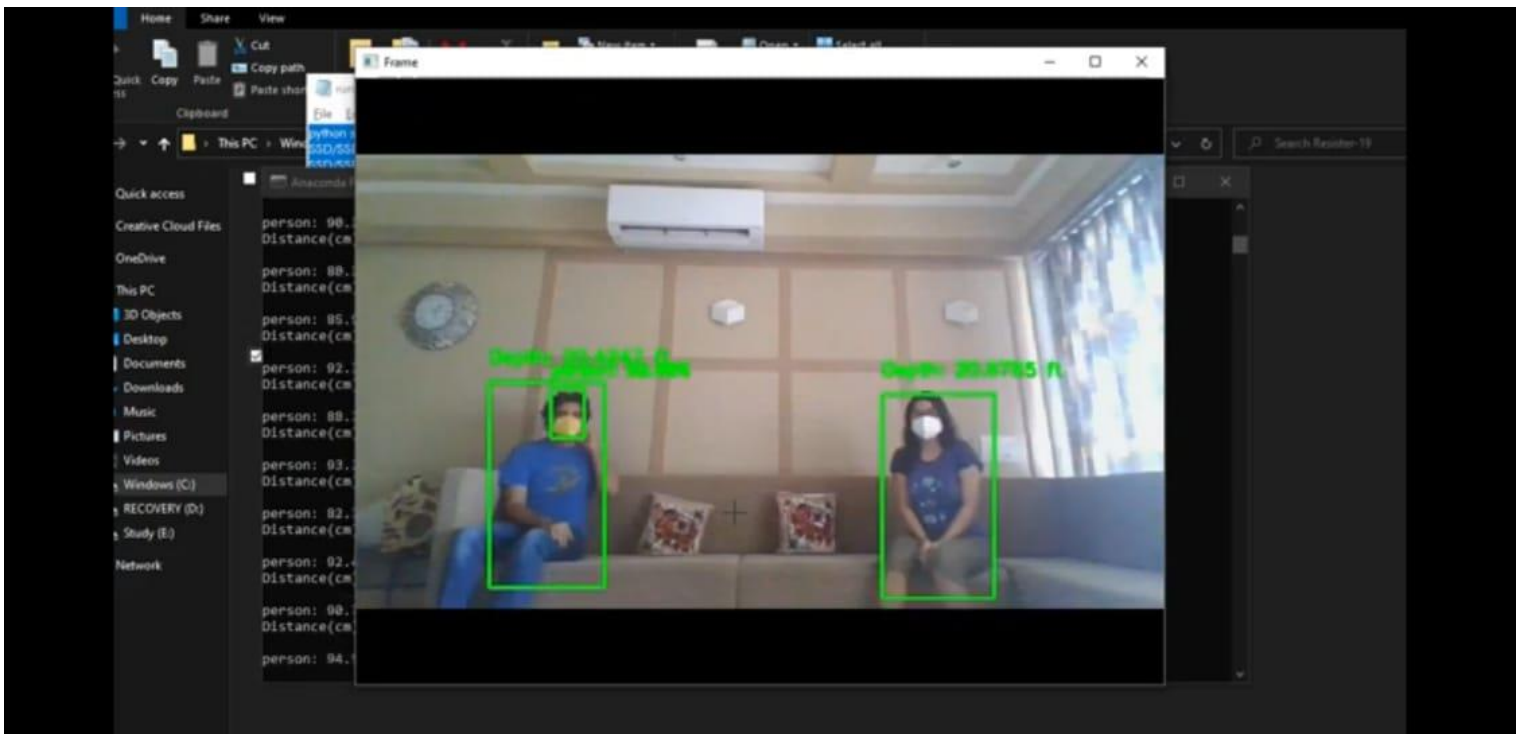

Fig.11 - Maintaining social distance with mask- no alarm 